\title{
Diagnostic and therapeutic difficulties in a patient with chronic recurrent multifocal osteomyelitis coexisting with ulcerative colitis
}

\author{
Beata Kołodziejczyk ${ }^{1}$, Agnieszka Gazda ${ }^{1}$, Elżbieta Hernik ${ }^{1}$, Izabela Szczygielska², Piotr Gietka ${ }^{1}$, \\ Iwona Witkowska ${ }^{1}$, Mateusz Płaza ${ }^{3}$ \\ ${ }^{1}$ Clinic of Developmental Age Rheumatology, National Institute of Geriatrics, Rheumatology and Rehabilitation, Warsaw, Poland \\ ${ }^{2}$ Rheumatology Clinic for Children, National Institute of Geriatrics, Rheumatology and Rehabilitation, Warsaw, Poland \\ ${ }^{3}$ Department of Radiology, National Institute of Geriatrics, Rheumatology and Rehabilitation, Warsaw, Poland
}

\begin{abstract}
Chronic non-bacterial osteomyelitis (CNO) is a rare autoinflammatory bone disease, affecting mainly children. CNO includes a broad clinical spectrum of symptoms and signs, from mild, limited in time, unifocal osteitis to severe, chronic, active or recurrent, multifocal osteomyelitis. In 2014 diagnostic criteria for CNO were proposed, the Bristol Criteria for the Diagnosis of Chronic Non-bacterial Osteitis, taking into account the clinical picture - location and number of inflammatory foci, characteristic changes on radiological examination (X-ray) and magnetic resonance imaging (MRI), C-reactive protein (CRP) concentration, and changes in bone biopsy.

The paper presents the case of a four-year-old boy in whom the diagnosis of multifocal osteomyelitis coexisting with ulcerative colitis was established. Attention was paid to the long diagnostic process of the disease, requiring in the first place differentiation with proliferative diseases. The choice of drugs was also a significant problem in the patient described in view of both intolerance of individual preparations and their ineffectiveness.
\end{abstract}

Key words: chronic non-bacterial osteomyelitis, chronic recurrent multifocal osteomyelitis, ulcerative colitis, sacroiliitis, magnetic resonance imaging.

\section{Introduction}

Chronic non-bacterial osteomyelitis (CNO) belongs to the group of autoinflammatory bone diseases and is a rare entity, affecting mainly children [1]. CNO was described for the first time in 1972 by Giedion [2].

In the group of autoinflammatory bone diseases the forms of genetically determined monogenic diseases occurring with non-infectious osteomyelitis of early onset are distinguished - Majeed syndrome (LPIN2 mutation), deficiency of interleukin 1 receptor antagonist (DIRA, ILIRN mutation) and pyogenic arthritis, pyoderma gangrenosum and acne syndrome (PAPA, PSTPIP1 mutation) [1]. The pathophysiology of the remaining CNO forms re- mains unknown - it seems complex, dependent on genetic and environmental factors. The main role is played by abnormal expression of cytokines in the cells of the inborn immune system, which leads to an imbalance between pro- and anti-inflammatory cytokines [1].

Chronic non-bacterial osteomyelitis includes a broad clinical spectrum of symptoms and signs, from mild, limited in time, unifocal osteitis to severe, chronic, active or recurrent, multifocal osteitis. This most severe form is defined as chronic recurrent multifocal osteomyelitis (CRMO) [1].

Chronic non-bacterial osteomyelitis occurs in every latitude [1]. In central Europe it is one of the most frequent autoinflammatory bone diseases. Onset of the 
disease under the age of two years is extremely rare [1]. CNO frequently coexists with other diseases, in the first place, such as inflammatory bowel diseases, arthritis including sacroiliitis, ankylosing spondylitis, skin lesions - acne, psoriasis including pustular palmoplantar psoriasis [1, 3, 4], and Sweet's syndrome [5]. Among adult patients with CNO, usually SAPHO (synovitis, acne, pustulosis, hyperostosis and osteitis) syndrome is diagnosed [1, 3].

In the course of CNO/CRMO, the pelvic bones, long bones (femur, tibia, fibula), vertebrae, clavicle, mandible, ribs, and calcaneum are most frequently involved [4]. The clinical symptoms and signs of osteitis include localized pain and/or oedema [4], sometimes elevated temperature, rarely skin reddening [1]. Pathological fractures may occur in the bones involved - this is mainly true of the vertebral column [1]. In the course of the disease, moderately elevated body temperature is sometimes observed [1]. The inflammatory parameter values are usually normal or slightly increased [4].

Imaging examinations are of key importance for the diagnosis of CNO/CRMO. Inflammatory bone lesions on radiological examination (X-ray) are of the character of osteolytic, osteosclerotic, hyperostotic foci, although at early stages of the disease the lesions may be invisible $[1,6-8]$. Magnetic resonance imaging (MRI) is a more sensitive imaging method $[1,4,6,8]$. Bone marrow oedema as a sign of osteitis on MRI is visible before the development of osteolytic and osteosclerotic lesions on X-rays and MRI. Magnetic resonance imaging of the whole body is particularly useful in the diagnosis of CNO. That examination makes it possible to assess the whole skeleton with respect to presence of foci of bone marrow oedema, and when performed during the treatment, it enables an assessment of disease activity [6-8]. Bone scintigraphy is also characterised by high sensitivity in detecting bone lesions, but low specificity is a limitation of that examination [4].

In unclear cases and when a unifocal lesion is present (with the exception of the clavicle), a bone biopsy is necessary in order to rule out a chronic infection, tu- mours or other systemic diseases. On histopathological examination of bones in CRMO patients, moderately intense infiltration by inflammatory cells is found - at an early stage of the disease, neutrophils predominate in the infiltration, while later, lymphocytes and plasma cells predominate, accompanied also by marrow cavity fibrosis [7, 9] and necrosis [8]. Bacterial cultures are always negative $[7,9]$.

In 2014, diagnostic criteria for CNO were proposed, the Bristol Criteria for the Diagnosis of Chronic Non-bacterial Osteitis, taking into account the clinical picture of the disease - location and number of inflammatory foci, characteristic changes on X-ray examination and MRI, C-reactive protein (CRP) concentration, and changes in bone biopsy [10]. These criteria are presented in Table I.

Depending on the course, $\mathrm{CNO}$ has been divided into the following subtypes:

- unifocal non-recurrent,

- unifocal recurrent,

- multifocal non-recurrent,

- multifocal recurrent [11].

The differential diagnosis includes infections (bacterial osteomyelitis, tuberculosis, etc.), malignancies (leukaemia, lymphoma, primary and secondary bone tumours) immunodeficiencies (e.g. IL-12 interferon deficiencies, which may be accompanied by mycobacterial infections), Langerhans cell histiocytosis ( $\mathrm{LCH}$ ) and other autoinflammatory bone diseases (Majeed syndrome, deficiency of interleukin receptor antagonist, pyogenic arthritis, pyoderma gangrenosum and acne syndrome) $[1,3,5]$.

In view of the rare occurrence of the disease and absence of randomised controlled studies, the treatment of CNO remains empirical [8]. The following are used: non-steroidal anti-inflammatory drugs (NSAIDs), corticosteroids, disease-modifying antirheumatic drugs (DMARDs), usually methotrexate or sulfasalazine, bisphosphonates, TNF- $\alpha$ blockers $[1,7,8]$.

According to the literature data, young patients with CNO may develop spondyloarthropathy in adulthood [12].

\section{Table I. Bristol diagnostic criteria for CRMO [10]}

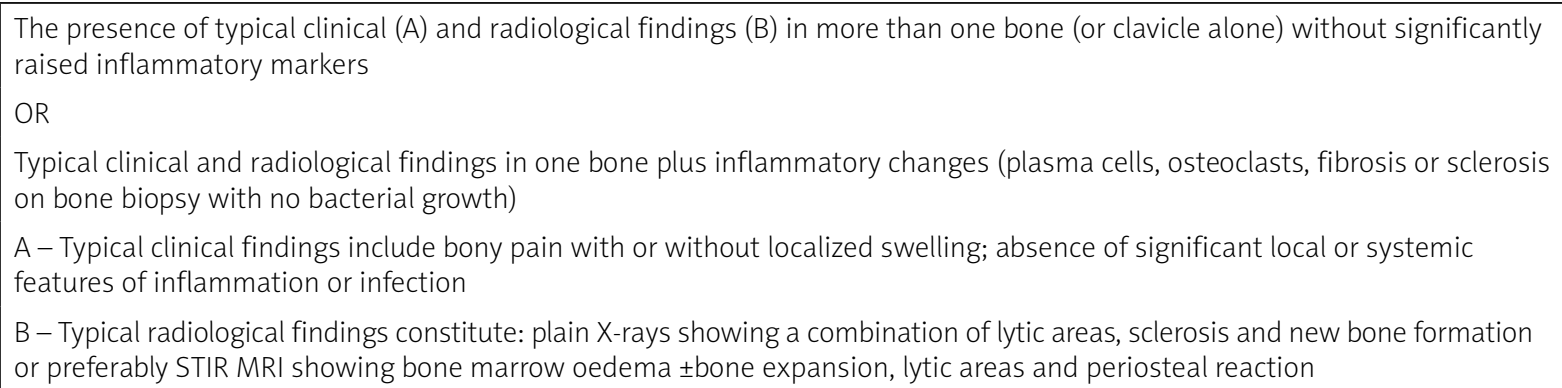




\section{Case report}

The described case concerns a boy aged four years and 6 months, with healthy parents, without any abnormalities during pregnancy and the perinatal period. In the medical history, frequent abdominal pain complaints and one-day diarrhoea (non-diagnosed manifestations) were found. In the period between May and August in 2015 the patient was treated for anaemia with iron supplementation, with an improvement. In the family medical history only psoriasis in the great-grandfather was described.

In August 2015 the patient suffered from a minor trauma of the lumbosacral region. From that time the boy had limping of the right lower limb and reported pain in the right buttock, and then in both buttocks and knees; no articular oedema was observed. In the period September-October 2015 ambulatory diagnostic procedures were conducted: laboratory tests - erythrocyte sedimentation rate (ESR) and C-reactive protein (CRP) - values were normal, in blood morphology slight anaemia and thrombocythemia were found; the rheumatoid factor (RF) was negative, $\mathrm{X}$-ray of the hip joints and $\mathrm{ul}$ trasound examination of the right hip were normal. The orthopaedic and neurological consultation did not reveal any problems and no deviations were recognized.

In November 2015 the patient was diagnosed for the first time in the Department of Developmental Age Rheumatology in National Institute of Geriatrics, Rheumatology and Rehabilitation in Warsaw. On medical examination no significant abnormalities were found, the joints showed no signs of arthritis, were normally mobile, the vertebral column, lumbosacral joint and but-

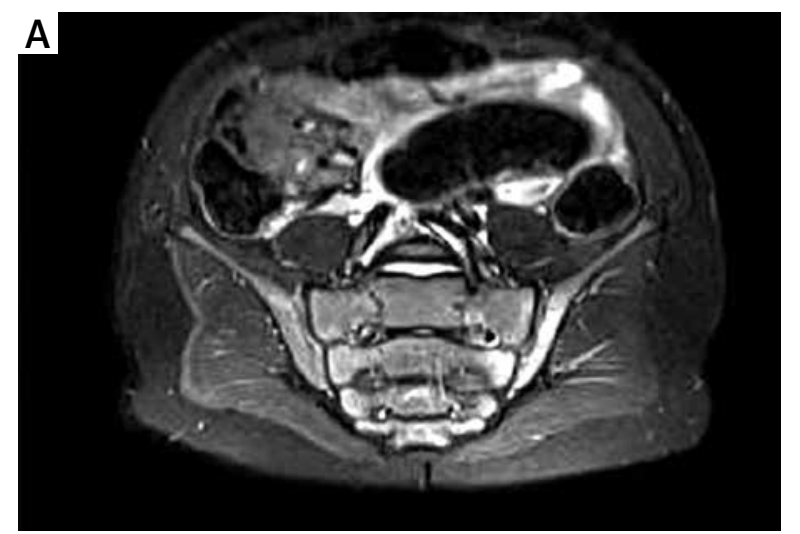

tock regions were not painful, the range of spinal movements was normal, flat-valgus feet and normal gait were observed. There were no abnormalities on neurological and ophthalmological examinations.

In laboratory test results the following were found: ESR $16 \mathrm{~mm} / \mathrm{h}$ (normal range 1-8 mm/h), CRP and procalcitonin levels were normal, in blood morphology slight thrombocythemia was found, ferritin concentration was decreased, lactate dehydrogenase ( $\mathrm{LDH}$ ) activity was increased, the serological tests for infectious factors gave negative results, the rheumatoid factor (RF) and anti-citrullinated peptide antibodies (ACPA) were negative, antinuclear antibodies (ANA) were weakly positive, in 1/80 titre, HLA-B27 antigen was absent. The tuberculin skin test was negative.

The chest X-ray and abdominal ultrasound examination were normal. On X-ray and MRI of the lumbar spine no abnormalities were found. On X-ray of the pelvis, left sacroiliitis at stage II was described, while the right sacroiliac joint and hip joints were normal. On the ultrasound examination of the hip and knee joints no changes were observed. The diagnostic procedures were extended to MRI of the sacroiliac joints and pelvis. The MRI examinations revealed bilateral sacroiliitis, the le-

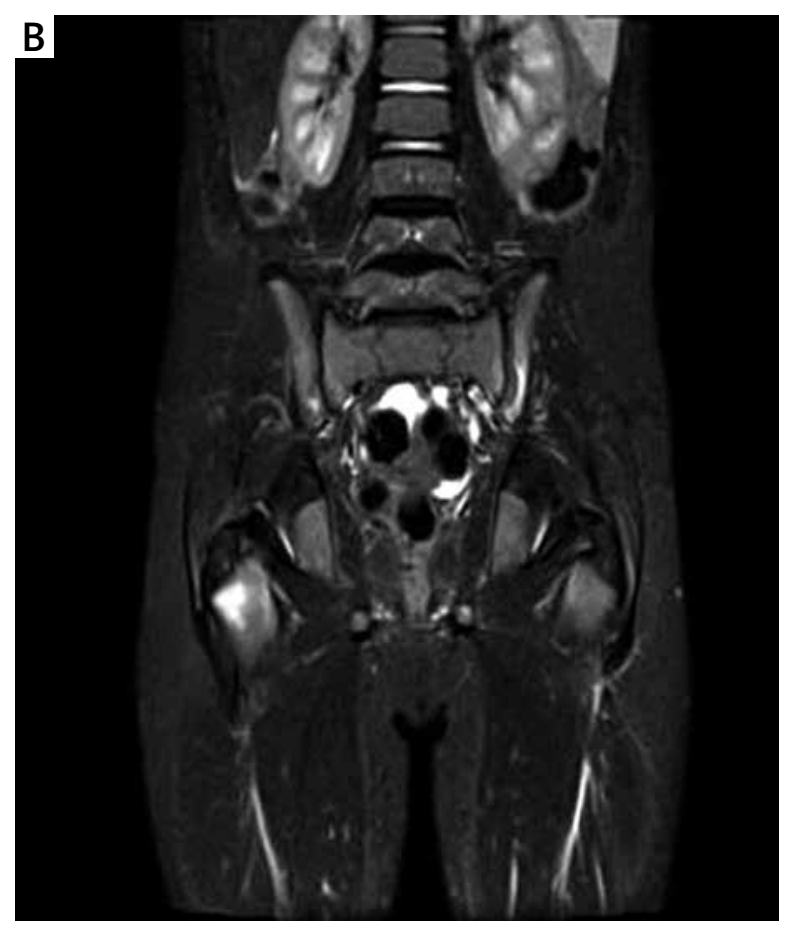

Fig. 1. A - MRI of the sacroiliac joints: regions of bone marrow oedema in both sacroiliac joints, the lesions are more pronounced in the left sacroiliac joint, the articular spaces are not widened, no exudate is seen, the contours of the left sacroiliac joint are irregular, those of the right one are smooth, signs of subchondral sclerotization, more pronounced on the left side. B - MRI of the pelvis: picture of bone marrow oedema, enhanced after gadolinium administration in the region of the greater trochanters of both femoral bones, the lesions are more pronounced on the right side. 
sions were more pronounced on the left side (Fig. 1A), and the picture of bone marrow oedema was evidently enhanced after gadolinium administration in the region of the greater trochanters of both femoral bones; no pathological changes were observed in the small pelvis organs (Fig. 1B).

In January 2016, whole body MRI (WB-MRI) was performed, which revealed additionally zones of bone marrow oedema in the distal metaphysis of the left tibial bone, in the right tarsal bone and in the first right metatarsal bone (Fig. 2).

A diagnosis of chronic recurrent multifocal osteomyelitis was considered. The patient met the Bristol Crite-

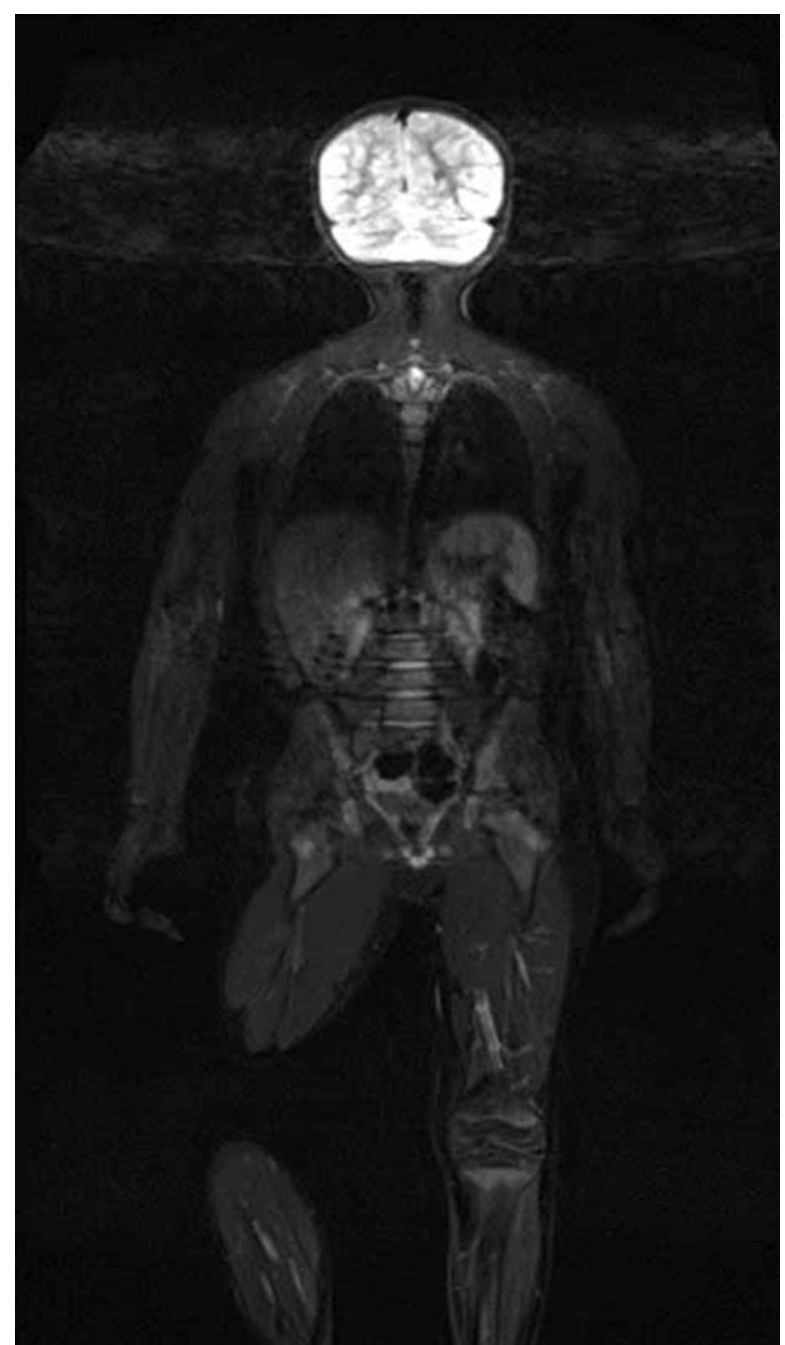

Fig. 2. MRI of the whole body: bone marrow oedema in the right tarsal bone, in the first metatarsal bone of the right foot, in the distal metaphysis of the left tibial bone, in the intertrochanteric regions of the femoral bones, in the sacral bone in the region of the left sacroiliac joint. ria for the Diagnosis of Chronic Non-bacterial Osteitis: typical clinical manifestations: ostealgia without local or systemic signs of inflammation or infection, typical changes on imaging examinations (MRI) (bone marrow oedema) and criterion 1 - changes in more than one bone, non-elevated CRP concentration. In the treatment, ibuprofen was administered under cover of a proton pump inhibitor.

In order to finally confirm chronic multifocal osteomyelitis, a wide differential diagnosis was performed. The orthopaedic spectrum diseases were excluded. The bone biopsy excluded a proliferative process.

In February and March the patient was diagnosed in the Oncologic Surgery Outpatient Clinic and Department of Surgery and Oncologic Surgery of Children and Adolescents, Institute of Mother and Child (IMC) in Warsaw. MRI of the lumbar spine was carried out; small foci of increased signal intensity, not undergoing contrast enhancement, were found in the sacral bone on the left side, in the left iliac bone, bilaterally in the pubic bones at the level of the pubic symphysis, in the subtrochanteric segments of the femoral bones, and a few inguinal lymph nodes of dimensions up to $10 \mathrm{~mm}$. The picture was assessed as equivocal - a proliferative process could not be ruled out with certainty. A right femoral bone biopsy was carried out and on histological examination no cells suspected of malignant proliferation were found. On control MRI of the pelvis, performed after the bone biopsy, small foci of increased signal intensity were found in locations as previously; no lesion progression was seen - the lesions were smaller. Besides that, no biopsy canal was observed within the lesion in the subtrochanteric region of the right femur (it was observed in the greater trochanter of the right femur), which suggested that the biopsy had not been taken from the planned site.

In the oncologic assessment, the picture of the disease indicated CRMO - this diagnosis was suggested by lower intensity of the lesions in the last MRI examination and regression of the pain complaints in the child after administration of non-steroidal anti-inflammatory drugs. A further oncologic follow-up was recommended - an MRI examination was planned after three months.

In April 2016 ibuprofen therapy was terminated and treatment with sulfasalazine was started. The examination results after the first period of treatment were normal. At two weeks after the beginning of sulfasalazine treatment, the child complained of abdominal pain and diarrhoea with blood, periodically was complaining of buttock pain, and limping was observed. In April 2016 the patient was diagnosed in the Department of Gastroenterology, Warsaw Medical University, where based on intestinal endoscopy and histological examination the diagnosis of ulcerative colitis (UC) was established. 

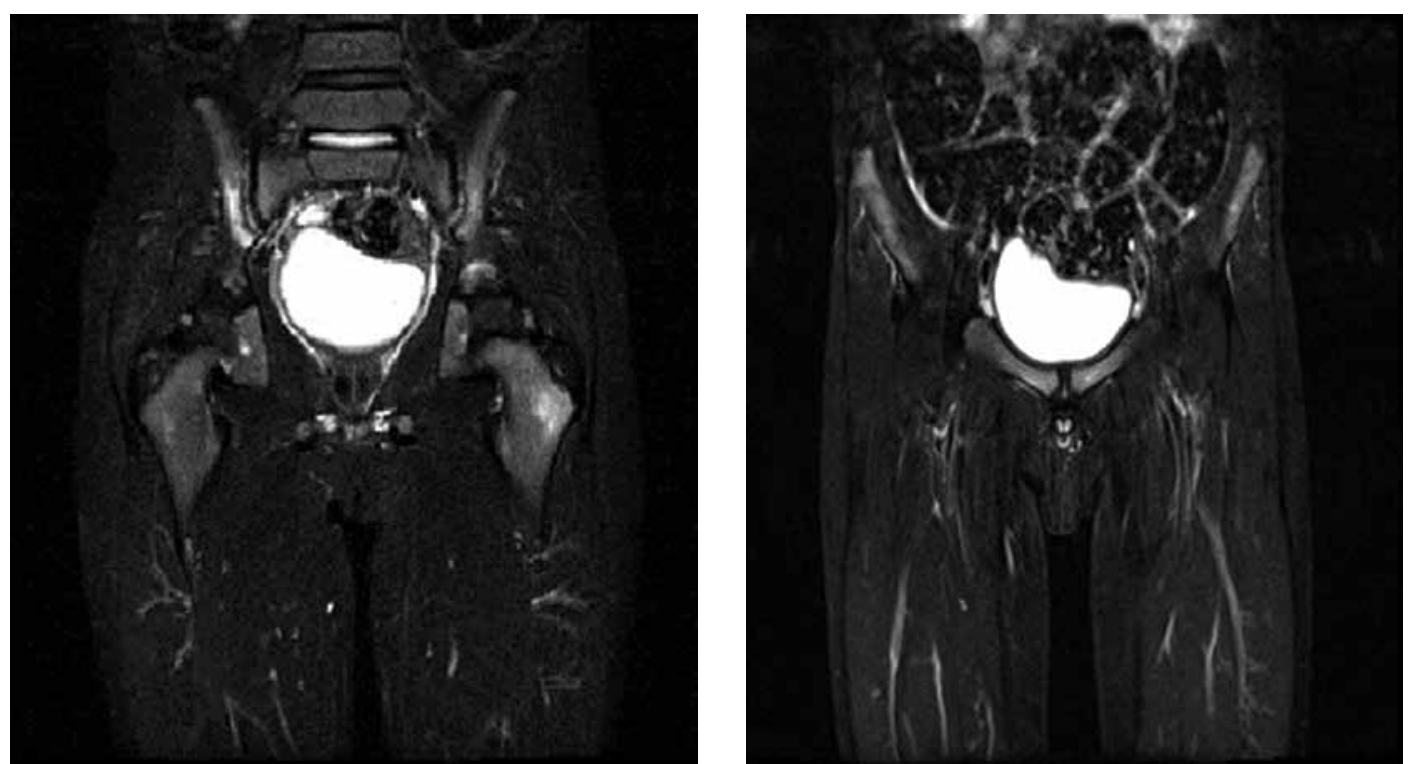

Fig. 3. Follow-up MRI of the pelvis: bone marrow oedema in the right sacroiliac joint, in the trochanter of the left femur, in the pubic symphysis bones, partial regression of the lesions in the trochanter of the right femur, complete regression in the left sacroiliac joint - migrating inflammatory lesions.

The treatment with sulfasalazine was terminated and mesalazine was administered. Then, in view of mesalazine intolerance, antibiotic therapy was given for three weeks.

In July 2016 MRI of the pelvis was carried out; in the right sacroiliac joint, the trochanter of the left femoral bone, in pubic symphysis bones inflammatory lesions were found, and partial regression of the lesions in the trochanter of the right femur, and complete regression of the lesions in the left sacroiliac joint were seen. The changes in MRI scan are presented in Figure 3.

Another oncological consultation took place and based on the follow-up and provided medical documentation a bone proliferative process was ruled out. The overall clinical picture suggested multifocal osteomyelitis coexisting with ulcerative colitis. A treatment of the underlying disease was recommended.

In September 2016 the treatment of UC with prednisone $1 \mathrm{mg} / \mathrm{kg}$ body weight daily for six weeks and azathioprine was administered. During the treatment with prednisone, the patient had no locomotor system complaints, was active, and no gastrointestinal symptoms or signs were observed.

After completion of the prednisone treatment, in October 2016 an intense pain occurred in the buttocks, hip joints, knee joints and feet. On medical examination, apart from slight contractures in the elbow joints, no changes of the peripheral joints were found, and also the vertebral column, sacroiliac joints and buttock region were normal. In laboratory tests, ESR was $13 \mathrm{~mm} / \mathrm{h}$,
CRP level was normal, and slight thrombocythemia and increased LDH activity were found. The ultrasound examinations of the elbow and hip joints and the left knee joint demonstrated no pathological changes. The whole body MRI examination revealed bone marrow oedema in the left femoral bone - similar to that in the examination of January 2016, regression of bone marrow oedema in the femur and calcaneus on the right side, and suspected bone marrow oedema in the right foot cuneiform bone. An X-ray examination of the feet no revealed any abnormalities, while MRI of the right foot revealed bone marrow oedema in the head of the $5^{\text {th }}$ metatarsal bone and in the base of the $1^{\text {st }}$ metatarsal bone. The treatment with azathioprine was continued.

In February 2017, in view of the persistence of pain complaints in the buttocks and peripheral joints, an MRI examination of the pelvis was carried out. In the examination the following were found: intensification of the bone marrow oedema compared with the previous examination, in the region of the femoral neck and greater trochanter on the left side and pubic bones near the pubic symphysis, and also bone marrow oedema in the neck and head of the femur and acetabular floor on the right side - a slightly different location of the lesions, and bone marrow oedema in the sacroiliac joints comparable with the previous examination, and synovial thickening in the sacroiliac joints. In the treatment, methotrexate $15 \mathrm{mg} / \mathrm{m}^{2}$ body surface area was given subcutaneously, instead of azathioprine. 
In view of the lack of clinical improvement, an increase of ESR value to $33 \mathrm{~mm} / \mathrm{h}$ with normal CRP level, in April 2017 a $10 \mathrm{mg}$ daily dose $(0.5 \mathrm{mg} / \mathrm{kg}$ body weight daily) of prednisone was added. During the treatment with prednisone in 0-7.5 mg daily doses, a clinical improvement was observed with regression of the pain complaints and ESR normalisation. After prednisone daily dose reduction to $<7.5 \mathrm{mg}$ the pain complaints in the buttocks and peripheral joints returned, and increased ESR was again found. On WB-MRI performed in January 2018, in the head of the right humeral bone, distal epiphysis of the radial bone bilaterally, epiphysis and metaphysis of the tibial and femoral bones forming the right knee joint, and distal epiphyses of the tibial bones new foci of bone marrow oedema were found, and lesions in the intertrochanteric regions of the femoral bones, sacroiliac joints and pubic bones were seen, comparable with the examination of November 2016. High doses of prednisone, $2 \mathrm{mg} / \mathrm{kg}$ body weight daily were given for six weeks. The doses were gradually reduced and the treatment with subcutaneous methotrexate was continued in a dose of $15 \mathrm{mg} / \mathrm{m}^{2}$ body surface area. During the treatment with high prednisone doses a clinical improvement was again observed. With a prednisone $10 \mathrm{mg}$ daily dose, a recurrence of pain of the buttocks and lower limbs and increase of ESR to $38 \mathrm{~mm} / \mathrm{h}$ were observed.

In March 2018 on the MRI of the sacroiliac joints bone marrow oedema and articular surfaces of the sacral and iliac bones were found in the anterior part, which was diagnosed as bilateral sacroiliitis. On MRI of the iliac joints a significant regression of bone marrow oedema in the bones forming the hip joints was observed. The treatment with prednisone was continued - with gradual dose reduction, methotrexate was given in a dose of $15 \mathrm{mg} / \mathrm{m}^{2}$ body surface area. In view of the predominant inflammatory changes in the sacroiliac joints, in April 2018 therapy with the TNF inhibitor adalimumab introduced. During the combined treatment with methotrexate and adalimumab, a clinical improvement - absence of locomotor system pain and normal inflammatory parameter values - was achieved. Disease recurrence after prednisone treatment termination was not observed. Table II presents a summary of changes in imaging studies over 2015-2018.

\section{Discussion}

It took months to establish a diagnosis in the described patient. The pain complaints in the buttocks and knees in a child patient made it necessary in the first place to rule out a local process. Post-traumatic lesions were excluded by imaging examinations of the lumbar spine, pelvis, peripheral joints and orthopaedic consultations.
No lesions suggesting an inflammatory process in the peripheral joints were found on either physical examination or imaging procedures. On the other hand, the presence of left sacroiliitis on X-ray and MRI examinations could have been a manifestation of juvenile idiopathic arthritis (JIA), i.e. arthritis of unknown aetiology, lasting at least six weeks and starting under the age of 16 years. In practice, however, isolated sacroiliitis without a preceding or coexistent peripheral arthritis occurs extremely rarely. Sacroiliitis in the course of JIA develops usually after several years of the disease course, i.e. at adolescence, most frequently in patients with a positive test result for HLA-B27 antigen. Both the young age and absence of inflammatory changes in the peripheral joints and absence of HLA-B27 antigen questioned the diagnosis of JIA. During further imaging diagnostic procedures, numerous foci of bone marrow oedema were found. Bone marrow oedema is a sign characteristic for CRMO, but is not very specific and requires a wide differential diagnosis.

Infectious processes were taken into account in the first place, such as bacterial osteomyelitis or tuberculosis, but absence of systemic manifestations, low inflammatory parameters, negative serological tests for infectious factors and negative tuberculin skin test spoke against them. Multifocal bone marrow oedema also made it necessary to rule out a proliferative diseases, such as leukaemia, lymphoma, primary and secondary bone tumours and histiocytosis, particularly in view of increased LDH activity observed in the patient. The normal bone marrow biopsy result and several-month-long oncologic follow-up made it possible to finally rule out a proliferative process. The occurrence of ulcerative colitis brought us closer to the diagnosis of CRMO.

Jansson et al. [9] described 89 patients with CNO; among them $61 \%$ of patients presented with at least two bone foci, $18 \%$ of patients had changes in pelvic bones and $22 \%$ had symmetrical bone lesions. Also $7 \%$ of patients had a diagnosis of inflammatory bowel diseases. Accelerated ESR was found in $82 \%$ of patients, elevated CRP in 15\%, and positive ANA was found in 33\% of patients [9].

The choice of the treatment in the described case is difficult because therapy required consideration of both locomotor system disease and gastrointestinal manifestations. During the treatment with sulfasalazine the patient developed UC symptoms The therapy with prednisone and azathioprine caused an evident improvement in UC symptoms, but pain complaints in the buttocks and peripheral joints, accelerated ESR and changes in the imaging examinations of the skeletal system continued. Introduction to methotrexate therapy did not allow the dose of glucocorticosteroids to be lowered. Only combination therapy of methotrexate with a TNF in- 
Table II. Summary of changes in MRI imaging studies

\begin{tabular}{|c|c|c|}
\hline $\begin{array}{l}\text { Stage of diagnostic } \\
\text { procedures/treatment }\end{array}$ & MRI of sacroiliac joints/pelvis & Whole body MRI \\
\hline $\begin{array}{l}\text { November 2015-March } 2016 \\
\text { CRMO suspected } \\
\text { Treatment: ibuprofen }\end{array}$ & $\begin{array}{l}\text { Bilateral sacroiliitis/bone marrow oedema of } \\
\text { the greater trochanters of both femoral bones }\end{array}$ & $\begin{array}{l}\text { Bone marrow oedema in the distal metaph- } \\
\text { ysis of the left tibial bone, in the right tarsal } \\
\text { bone, in the first right metatarsal bone }\end{array}$ \\
\hline $\begin{array}{l}\text { April 2016-July } 2016 \\
\text { UC and CRMO diagnosis } \\
\text { Treatment: sulfasalazine, } \\
\text { mesalazine, antibiotic therapy, } \\
\text { prednisone, azathioprine }\end{array}$ & $\begin{array}{l}\text { Bone marrow oedema in right sacroiliac joint, } \\
\text { trochanter of the left femoral bone, in pubic } \\
\text { symphysis bones, and partial regression of } \\
\text { the lesions in the trochanter of the right } \\
\text { femur, and complete regression of the lesions } \\
\text { in the left sacroiliac joint }\end{array}$ & \\
\hline $\begin{array}{l}\text { September 2016-January } 2017 \\
\text { Treatment: azathioprine }\end{array}$ & & $\begin{array}{l}\text { Bone marrow oedema in the left femoral } \\
\text { bone, in the cuneiform bone of the right } \\
\text { foot, regression of bone marrow oedema in } \\
\text { the femur and calcaneus on the right side }\end{array}$ \\
\hline $\begin{array}{l}\text { February } 2017-\text { December } 2017 \\
\text { Treatment: methotrexate, } \\
\text { prednisone } 0.5 \mathrm{mg} / \mathrm{kg} \text { body } \\
\text { weight daily }\end{array}$ & $\begin{array}{l}\text { Intensification of the bone marrow oedema } \\
\text { of the femoral neck and greater trochanter on } \\
\text { the left side and pubic bones near the pubic } \\
\text { symphysis, and also bone marrow oedema } \\
\text { in the neck and head of the femur and } \\
\text { acetabular floor on the right side - a different } \\
\text { location of the lesions, and bone marrow } \\
\text { oedema in the sacroiliac joints }\end{array}$ & \\
\hline $\begin{array}{l}\text { January } 2018 \text {-February } 2018 \\
\text { Treatment: methotrexate, } \\
\text { prednisone }-2 \mathrm{mg} / \mathrm{kg} \text { body } \\
\text { weight daily, dose reduction } \\
\text { over six weeks }\end{array}$ & & $\begin{array}{l}\text { New foci of bone marrow oedema in the } \\
\text { head of the right humeral bone, distal } \\
\text { epiphysis of the radial bone bilaterally, } \\
\text { epiphysis and metaphysis of the tibial and } \\
\text { femoral bones forming the right knee joint, } \\
\text { distal epiphyses of the tibial bones, and } \\
\text { persistence of the lesions in the intertro- } \\
\text { chanteric regions of the femoral bones, } \\
\text { sacroiliac joints and pubic bones }\end{array}$ \\
\hline $\begin{array}{l}\text { March } 2018 \text { - until the present } \\
\text { Treatment: methotrexate, } \\
\text { prednisone - termination of } \\
\text { treatment, adalimumab }\end{array}$ & $\begin{array}{l}\text { Bone marrow oedema and uneven articular } \\
\text { surfaces of the sacral and iliac bones were in } \\
\text { the anterior part - bilateral sacroiliitis, } \\
\text { significant regression of bone marrow } \\
\text { oedema in the bones forming the hip joints }\end{array}$ & \\
\hline
\end{tabular}

hibitor allowed the desired effect to be achieved. Other studies confirmed that treatment with corticosteroids, sulfasalazine, NSAID with methotrexate and in refractory cases anti-TNF therapy is the most effective $[13,14]$.

The patient requires further rheumatologic follow-up. Sacroiliitis, predominating in the clinical picture, may accompany CRMO, but spondyloarthropathy development cannot be ruled out in the patient. It is possible that the patient, with an established diagnosis of chronic recurrent multifocal osteomyelitis coexistent with ulcerative colitis, at adult age may develop symptoms of peripheral arthritis in the course of ulcerative colitis.

\section{Conclusions}

Chronic non-bacterial osteomyelitis includes a broad clinical spectrum of symptoms and signs, from mild, lim- ited in time, unifocal osteitis to severe, chronic, active or recurrent, multifocal osteomyelitis. The Bristol Criteria for the Diagnosis of Chronic Non-bacterial Osteitis, proposed in 2014, take into account the clinical picture (location and number of inflammatory foci), characteristic changes on radiological examination and magnetic resonance imaging, laboratory test results such as C-reactive protein concentration and changes in bone biopsy [10].

The present case showed the long diagnostic process, requiring in the first place differentiation with proliferative diseases. The final diagnosis of CNO/CRMO takes time and multi-specialist cooperation. There are no specific laboratory tests or unambiguous imaging tests confirming the diagnosis. However, whole body $M R I$ is highly effective in diagnosis of CRMO, which is confirmed in the literature [15]. Effective CRMO treat- 
ment, especially with another coexisting inflammatory disease, may be dependent on individual sensitivity to the drugs and related to disease activity. NSAIDs, sulfasalazine, methotrexate and TNF inhibitors as treatment options alone and in combined therapy are now being discussed. However, there are no prospective clinical trials to determine optimal CRMO therapy.

The authors declare no conflict of interest.

\section{References}

1. Hofmann SR, Kapplusch F, Girschick HJ, et al. Chronic Recurrent Multifocal Osteomyelitis (CRMO): Presentation, Pathogenesis, and Treatment. Curr Osteoporos Rep 2017; 15: 542-554.

2. Giedion A, Holthusen W, Masel LF, Vischer D. Subacute and chronic "symmetrical" osteomyelitis. Ann Radiol (Paris) 1972; 15: 329-342.

3. Thakur U, Blacksin M, Beebe K, et al. Synovitis, Acne, Pustulosis, Hyperostosis and Osteitis (SAPHO) and chronic recurrent multifocal osteomyelitis (CRMO): Role of imaging in diagnosis. Radiography 2012; 18: 221-224.

4. Borzutzky A, Stern S, Reiff A, et al. Pediatric chronic nonbacterial osteomyelitis. Pediatrics 2012; 130: 1190-1197.

5. Tlougan BE, Podjasek JO, Haver J, et al. Chronic recurrent multifocal osteomyelitis (CRMO) and synovitis, acne, pustulosis, hyperostosis, and osteitis (SAPHO) syndrome with associated neutrophilic dermatoses: a report of seven cases and review of the literature. Pediatr Dermatol 2009; 26: 497-505.
6. von Kalle T, Heim N, Hospach T, et al. Typical patterns of bone involvement in whole-body MRI of patients with chronic recurrent multifocal osteomyelitis (CRMO). Rofo 2013; 185: 655-661.

7. Taddio A, Ferrara G, Insalaco A, et al. Dealing with Chronic Non-Bacterial Osteomyelitis: a practical approach. Pediatr Rheumatol Online J 2017; 15: 87.

8. Zhao Y, Dedeoglu F, Ferguson PJ, et al. Physicians' Perspectives on the Diagnosis and Treatment of Chronic Nonbacterial Osteomyelitis. Int J Rheumatol 2017; 2017: 7694942.

9. Jansson A, Renner ED, Ramser J, et al. Classification of Non-Bacterial Osteitis: Retrospective study of clinical, immunological and genetic aspects in 89 patients. Rheumatology 2007; 46: 154-160.

10. Ramanan AV, Roderick M, Shah R, et al. Proposal of the Bristol Criteria for the Diagnosis of Chronic Non-bacterial Osteitis From a Cohort of 41 Patients. Arthritis Rheum 2014; 66: S107.

11. Girschick HJ, Raab P, Surbaum S, et al. Chronic non-bacterial osteomyelitis in children Ann Rheum Dis 2005; 64: 279-285.

12. Vittecoq O, Said LA, Michot C, et al. Evolution of chronic recurrent multifocal osteitis toward spondylarthropathy over the long term. Arthritis Rheum 2000; 43: 109-119.

13. Marangoni RG, Halpern AS. Chronic recurrent multifocal osteomyelitis primarily affecting the spine treated with anti-TNF therapy. Spine (Phila Pa 1976) 2010; 35: E253-256.

14. Batu ED, Gulhan B, Topaloglu R, Ozen S. A proposed treatment scheme for chronic recurrent multifocal osteomyelitis (CRMO): a case series of nine patients. Pediatr Rheumatol Online J 2014; 12 (Suppl 1): P239.

15. Beck C, Morbach H, Beer M, et al. Chronic nonbacterial osteomyelitis in childhood: prospective follow-up during the first year of anti-inflammatory treatment. Arthritis Res Ther 2010; 12: R74. 